\title{
Yersinia enterocolitica
}

\section{Stellungnahmen des Arbeitskreises Blut des Bundesministeriums für Gesundheit}

De er Arbeitskreis Blut des Bundesministeriums für Gesundheit gibt als nationales Beratungsgremium Stellungnahmen zu neuartigen Erregern ab, bewertet neue Erkenntnisse zu bekannten Erregern und erarbeitet entsprechende Empfehlungen für die Fachöffentlichkeit. Diese Serie von Stellungnahmen zu einzelnen Erregern werden als Zusammenfassung des aktuellen Wissensstandes veröffentlicht, speziell unter transfusionsmedizinisch relevanten Aspekten (Bundesgesundhbl. 41, 2 (1998), 53). Frühere Beiträge befaßten sich mit der Creutzfeldt-Jakob-Erkrankung, dem Parvovirus B19 und dem GB-Virus Typ C (Hepatitis-G-Virus) (Bundesgesundhbl. 41, 2 (1998) 78-90) und HTLVI/-II, (Bundesgesundhbl. 41, (1998) 512).

\section{Wissensstand über den Erreger}

\subsection{Erregereigenschaften}

Das gramnegative Bakterium Yersinia enterocolitica ( $Y$. enterocolitica) ist im Blutspendewesen von besonderer $\mathrm{Be}$ deutung. Der Erreger wurde wiederholt, wenn auch insgesamt sehr selten, als Ursache transfusionsassoziierter Septikämien mit überwiegend tödlichem Ausgang identifiziert. Fast ausschließlich gingen diese Ereignisse von Erythrozytenkonzentraten aus.

Die Spezies Y. enterocolitica gehört zusammen mit Yersinia pestis und Yersinia pseudotuberculosis und weiteren nicht pathogenen Yersinien zur Gattung Yersinia. Yersinien gehören zu den Enterobacteriaceae, einer großen Familie gramnegativer Bakterien.

Die lipidhaltige Doppelmembran, aus der die Zellwand gramnegativer Bakterien aufgebaut ist, spielt beim Ablauf der transfusionsassoziierten Infektionen mit diesen Erregern eine herausragende Rolle. Die in dem äußeren Membrananteil eingebetteten Lipopolysaccharide (LPS, Endotoxin) können, sofern sie in die Blutbahn freigesetzt werden, den Endotoxinschock auslösen. Andererseits ist die äußere Membran Ziel direkter Angriffe der Immunabwehr über Komplementsystem, Antikörper und Phagozyten, die eine Abtötung des Bakteriums ermöglichen.

Innerhalb der Spezies Y. enterocolitica werden die Stämme nach Serogruppen- bzw. Biotypenzugehörigkeit unterteilt. In Europa gelten die Serovare O:3, O:9 und $0: 5,27$ in der Humanmedizin als bedeutsam, in Nordamerika die Serovare O:3, O:8, O:13, O:20 und O:21. Das Serovar O:3 konnte über längere Zeit nur in Europa nachgewiesen werden. Inzwischen wird es auch in Nordamerika ge- funden, während $0: 8$, der häufigste der nordamerikanischen Vertreter, in Europa bisher sehr selten nachgewiesen wurde. Der jeweiligen Serovarietät bzw. dem Biotypen werden auch Pathogenitätsmerkmale zugeordnet. So gilt das Serovar 0:8 im allgemeinen als virulenter verglichen mit den Serovaren O:3 oder O:9, während Stämme des Biotyps IB nicht als humanpathogen gelten $[1,2]$.

Yersinien sind psychrophil, d.h. sie vermehren sich auch bei $0-4^{\circ} \mathrm{C}[3]$. Diese Eigenschaft wird bei der sogenannten Kälteanreicherung zur selektiven Keimvermehrung in Stuhlproben ausgenutzt. Ebenso können sich Yersinien in kühlgelagerten Materialien, wie Lebensmitteln oder den hier im Mittelpunkt stehenden Erythrozytenkonzentraten, vermehren und, wenn lange genug aufbewahrt, darin hohe Keimzahlen erreichen (s.u.).

Viele Bakterien verfügen über spezifische Mechanismen zur Aufnahme von Eisen aus ihrer Umgebung. Die Fähigkeit der verschiedenen Serotypen von Y. enterocolitica, die für ihre Vermehrung notwendigen Eisenionen in unterschiedlicher Weise aufzunehmen, ist ausführlich untersucht worden [4]. Bei den im Plasma vorliegenden extrem niedrigen Konzentration von etwa $10^{-15}$ $\mathrm{M}$ freiem Eisen sind einige Yersinien in der Lage, ihren Bedarf von etwa $10^{-6} \mathrm{M}$ 
Eisen zu befriedigen. Die extrem niedrige Konzentration an freiem Eisen geht auf intra- und extraplasmatische Proteine wie Transferrin, Laktoferrin und Ferritin zurück, die eine hohe Affinität für (dreiwertige) Eisenionen aufweisen. Diese umfangreichen Vorkehrungen des Wirtsorganismus werden als eine Abwehrmaßnahme gegen in Gewebe und Blutbahn eindringende Erreger verstanden. Das von Y. enterocolitica dagegen aufgebotene System besteht in kompletter Ausfertigung aus zwei Komponenten, den Siderophoren und den Siderophorenrezeptoren. Die Siderophoren werden vom Bakterium in die Umgebung abgegeben und binden dort als komplexbildende Moleküle $(<1 \mathrm{kD})$ die Eisenionen $\left(\mathrm{Fe}^{3+}\right)$ in Konkurrenz zu den eisenbindenden Proteinen des Wirtsorganismus. Der somit entstehende EisenSiderophorenkomplex wird mit Hilfe der Siderophorenrezeptoren, die auf der Oberfläche der Bakterien lokalisiert sind, in das Zellinnere eingeschleust [5].

Nicht alle Y. enterocolitica-Stämme besitzen beide Komponenten. Während beispielsweise Angehörige der Serovare O:8 und O:21 mit dem kompletten System ausgestattet sind, besitzen Angehörige der Serovare O:3, O:9, O:5,27, O:1,2,3 und O:20 nur die Rezeptoren. Bezüglich der natürlichen Infektion ist diese unterschiedliche Ausstattung in der bei Serovar 0:3 gegenüber Serovar O:8 beschriebenen geringeren Virulenz, die im Mausmodell gemessen wurde (s.o.), wiederzuerkennen. Wurden die fehlenden Siderophoren der O:3-Stämme von außen durch Siderophoren anderer Mikroorganismen ersetzt, konnte ihre geringere Virulenz angehoben werden. Durch Zugabe von Desferrioxamin, einem von Streptomyceten gebildeten Siderophorin, ließ sich in infizierten Mäusen das Wachstum von Keimen des Serovar O:8 nur geringfügig (Faktor 10-100), die der Serovare 0:3 hingegen um das $10^{5}$ fache steigern. Kongruent dazu liegen klinische Beobachtungen vor, nach denen bei Patienten unter Therapie mit Desferrioxamin, das zur Ausschwemmung von pathologisch erhöhtem Eisen eingesetzt wird, vermehrt schwer verlaufende Infektionen mit Y. enterocolitica, Serovar O:3, auftraten. Hämatochromatosen, chronische Niereninsuffizienz, hämolytische Anämien und auch häufige Transfusionen von erythrozytenhaltigen Konserven erhöhen das verfügbare Eisen im Plasma. Mit hämolytischen Anämien und wiederholten Transfusionen erhöht sich im Blut der Gehalt an Hämoglobin, das aus den zerfallenden Erythrozyten freigesetzt wird. Das aus dem Hämoglobin stammende Hämin kann von Y. enterocolitica ähnlich den Siderophoren durch eigene Rezeptoren aufgenommen werden; die nicht mit Siderophoren ausgestatteten Bakterien haben damit die ihnen fehlende Eisenquelle [4].

Neben den die Eisenaufnahme bestimmenden Faktoren wurden bei Yersinien weitere bedeutende Virulenzfaktoren beschrieben. Sie sind chromosomal- und plasmidkodiert. Die vom Plasmid kodierten Faktoren werden fast ausschließlich bei $37^{\circ} \mathrm{C}$ exprimiert. Dieses $70 \mathrm{~kb}$ große Plasmid kann bei der Invitro-Vermehrung leicht verloren gehen, wenn sie bei Temperaturen über $30^{\circ} \mathrm{C}$ erfolgt. Daher muß bei In-vitro-Experimenten in den verwendeten Stämmen die Präsenz des Plasmids explizit nachgewiesen sein und andererseits die Ausgangstemperatur, bei der die Stämme unmittelbar vor dem Experiment gehalten wurden, als wichtige Bedingung des Experimentes beachtet werden. Die chromosomalen Virulenzfaktoren wer- den bei $37^{\circ} \mathrm{C}$ teils an-, teils abgeschaltet. Tabelle 1 enthält eine Aufstellung wichtiger Pathogenitätsfaktoren und ihre Temperaturabhängigkeit.

Die unterschiedliche Struktur der Lipopolysaccharide (LPS) in Abhängigkeit von der Temperatur wirkt sich in folgender Weise aus: Die rauhe, kurzkettige Form, die bei $37^{\circ} \mathrm{C}$ gebildet wird, bleibt in der Zellmembran integriert. Die glatte, langkettige Form, die bei $\leq 25^{\circ} \mathrm{C}$ gebildet wird, kann sich von der Membran ablösen und als freies Endotoxin wirksam werden. Dies ist mit eine Erklärung dafür, weshalb bei transienten Yersiniämien (bei der Körpertemperatur von $37^{\circ} \mathrm{C}$ ) mit relativ niedrigen Keimzahlen die für eine gramnegative Sepsis typische Symptomatik fehlt. Vermehren sich hingegen die Keime in Erythrozytenkonzentraten bei den niedrigen Aufbewahrungstemperaturen von ca. $4^{\circ} \mathrm{C}$, kommt es zu hohen Konzentrationen an freiem langkettigen LPS, das im Transfusionsempfänger schließlich das volle Bild eines akuten lebensbedrohenden Endotoxinschocks auslöst (Mündliche Mitteilung J. Heesemann).

Die vom Plasmid gesteuerten Faktoren verhelfen dem Bakterium bei $37^{\circ} \mathrm{C}$ dazu, die unspezifische Abwehr des befallenen Organismus zu überwinden. Das zeigt sich an verrringerter Komplement-Aktivierung über den „alternati-

\begin{tabular}{|c|c|c|c|}
\hline \multirow[t]{2}{*}{ Lokalisation } & \multirow[t]{2}{*}{ Pathogenitätsfaktor } & \multicolumn{2}{|c|}{ Exprimiert bei } \\
\hline & & $37^{\circ} \mathrm{C}$ & $\leq 25^{\circ} \mathrm{C}$ \\
\hline \multirow[t]{5}{*}{ Chromosom } & Struktur der Lipopolysaccharide (Kolonieform) & kurzkettig (rauh) & langkettig (glatt) \\
\hline & Zelluläre Morphologie & pleomorph & kokkoid \\
\hline & Adhärenz an phagozytierende Zellen & schwach & stark \\
\hline & Anwesenheit von Geißeln (Motilität) & nein & ja \\
\hline & Invasin (inv) & gering & stark \\
\hline \multirow[t]{6}{*}{ Plasmid } & Spezifische Proteine der äußeren Membran & ja & nein \\
\hline & Hydrophobizität der Zelloberflächen & ja & nein \\
\hline & Kalziumabhängigkeit des Wachstums & ja & nein \\
\hline & Resistenz gegen Serumbakterizidie ${ }^{*}$ & ja & nein \\
\hline & Resistenz gegen Phagozytose & ja & nein \\
\hline & Resistenz gegen intrazelluläre Abtötung & ja & nein \\
\hline
\end{tabular}

${ }^{*}$ Kann je nach Serotyp variieren (z.B. ist Serotyp 0:3 bei $\leq 25^{\circ} \mathrm{C}$ serumresistenter als 0:8) 
ven Weg“ (erhöhte Serumresistenz), der Phagozytose-Resistenz und verminderter intrazellulärer Abtötung der Keime durch Makrophagen. Die erst durch die Infektion ausgelöste spezifische Immunabwehr kann die Elimination der Keime herbeiführen. Es wird spekuliert, daß $Y$. enterocolitica, in langlebige Zellen eingedrungen, über lange Zeit im Körper persistieren kann und so zur Ursache „unerklärlicher" Bakteriämien wird [6].

\section{Die Vermehrung von Yersinia enterocolitica in Erythrozytenkonzentraten}

Y. enterocolitica hatte in den regelrecht gelagerten Erythrozytenkonzentraten, die zu Septikämien führten, eine Keimzahl von $10^{8}-10^{9} / \mathrm{ml}$ erreicht. Nach den heutigen Vorstellungen liegt der Keim in der Konserve aufgrund einer unerkannten Bakteriämie des Spenders zunächst im Bereich von 10-100 CFU (Colony Forming Units)/ml vor. Erst nach Ablauf von zwei bis drei Wochen beginnt die rasche Vermehrung des Keimes. Die diesem Geschehen zugrunde liegenden Vorgänge sind nicht vollständig geklärt. Es scheint jedoch, daß die besonderen Eigenschaften von Y. enterocolitica, und insbesondere die der Serovare O:3, O:9 u.ä., bei der Entwicklung hoher Keimzahlen in den kontaminierten Konserven zusammenwirken (Vergl. [7]). Wie die Abläufe bei der Herstellung und Lagerung der Erythrozytenkonzentrate dazu beisteuern, läßt sich in der nachfolgenden Weise darstellen, auch wenn nicht alle damit implizierten Prozesse belegt sind.

Am Anfang steht die klinisch nicht erkannte Bakteriämie. Sie ist deshalb kaum festzustellen, weil die Keimdichte sehr niedrig ist, das kurzkettige LPS wenig pathogen ist und nur in geringem Umfang aus der outer membrane freigesetzt wird. Die bei einer akuten Yersinien-Infektion erwartete Durchfallssymptomatik kann ausbleiben, unbeachtet Tage vor der Spende oder erst nach der Spende auftreten. In zwei Drittel der Yersinia-assoziierten Zwischenfälle ließ sich überhaupt keine abdominale Symptomatik ermitteln (Vgl. [8]). Der Ur- sprung der Keime kann auch außerhalb des Bauchraumes vermutet werden, etwa in intrazellulärer Form in den Geweben persistierend (Vgl. [6]).

Bei der Abkühlung der als Vollblutkonserve vorliegende Spende verliert Y. enterocolitica die plasmidkodierten Eigenschaften. Die neu aktivierten Eigenschaften, die chromosomal kodiert sind, ermöglichen dem Bakterium das Eindringen in phagozytierende Zellen. Extrazellulär verbleibende Keime können über Komplement-Aktivierung eliminiert werden, die intrazellulären Erreger überleben. Entscheidend ist, daß für die Abtötung bzw. Aufnahme in die Phagozyten hinreichend Zeit bleibt.

Bei der folgenden Komponententrennung werden die Erythrozyten in plasmaarmes Medium überführt und anschließend auf die Lagerungstemperatur von $4 \pm 2{ }^{\circ} \mathrm{C}$ abgekühlt. Komplement, wenn noch in wirksamen Mengen vorhanden, ist jetzt kaum mehr aktivierbar. Nach ca. einer Woche beginnen die Leukozyten in größerem Umfang zu zerfallen und die noch lebensfähigen Yersinien freizugeben. Der gleichzeitige stetige Zerfall kleiner Mengen von Erythrozyten versorgt die Keime mit Eisen, das im Hämin in verwertbarer Form zur Verfügung steht. Gegen Ende der zweiten Lagerungswoche beginnt gewöhnlich die mikrobiologisch nachweisbare Vermehrung der Bakterien, die dann in der dritten Woche hohe Konzentrationen erreichen und große Mengen an LPS freisetzen können.

Aus In-vitro-Versuchen ging hervor, daß sich der Serotyp O:8 in experimentell inokulierten Erythrozytenkonzentraten unter diesen Bedingungen deutlich geringer vermehren kann als der Serotyp O:3. Dies bestätigt die anderweitig beobachtete ausgeprägte Serumresistenz von $\mathrm{O}: 3$ unter $25^{\circ} \mathrm{C}$ [9] und ist in Kongruenz mit dem hohen Anteil des O:3-Serotyps an transfusionsassoziierten Sepsisfällen.

\subsection{Infektion und Infektionskrankheit}

Das klinische Spektrum der durch die humanpathogenen $Y$. enterocolitica verusachten Infektionen umfaßt im wesentlichen
Gastrointestinale Erkrankungen: Enterokolitis oder Pseudoappendizitis

Septikämie: Bevorzugt in immungeschwächten und an Eisenüberladung leidenden Patienten, die mit Desferrioxamin behandelt werden oder transfusionsassoziiert (s. 3.3)

D Metastatische Ansiedlung mit Abszessbildung oder Organerkrankung in Haut, Lunge, Endocard usw. nach Septikämien

D Postinfektiöse Folgen (Sequelae) wie sie auch im Gefolge anderer bakterieller Infekte auftreten können, wie Arthritis, Myokarditis, Glomerulonephritis, Erythema nodosum (hier auf Träger des HLA-B27-Antigens beschränkt)

Bei der peroralen Infektion können sich die Keime im ileozökalen Bereich festsetzen und dort Ulzera verursachen, häufig begleitet von transienter Bakteriämie. Es kommt entweder zu spontaner Abheilung der Ulzera oder auch zu septikämischem Verlauf. Bei Kleinkindern kann sich die Yersinieninfektion zu einer schweren Enterokolitis entwickeln, die lebensbedrohlich werden kann. Rektale Blutungen und Darmperforation können auftreten. Die Enterokolitis der älteren Kinder und der Erwachsenen äußert sich gewöhnlich mit Durchfall, Fieber und Leibschmerzen, mit Fokussierung auf den rechten unteren Quadranten des Abdomens unter Ausbildung einer pseudoappendizitischen Symptomatik, die durch eine Entzündung der lokalen Lymphknoten ausgelöst wird. Die Symptomatik kann jedoch auch nur angedeutet bis inapparent sein. Klinisch ist die Symptomatik beim Erwachsenen kaum von der Vielzahl anderer in Frage kommender „banaler“ gastrointestinaler Infekte abzugrenzen. Bei dieser Symptomatik wird eine mikrobiologische Diagnostik selten eingeleitet.

Der Erreger kann nach Abklingen der Symptome noch weiter (zwei bis sechs Wochen) mit dem Stuhl ausgeschieden werden [10]. Diskutiert wird, ob der Keim darüber hinaus in den Geweben noch langwährend persistiert [6]. 


\subsection{Epidemiologie}

Y. enterocolitica ist weltweit verbreitet, jedoch unter Bevorzugung gemäßigter bis kühlerer Klimazonen. Innerhalb der Serotypen sind die Typen 0:3 und 0:9 in (Nord)Europa am weitesten verbreitet, in Nordamerika O:3 und O:8. Der zunächst nur in Europa aufgetretene Serotyp 0:3 hat sich erst jüngst auf Nordamerika ausgebreitet. Der als besonders virulent angesehene Serotyp O:8 ist in Deutschland bislang noch nicht identifiziert worden, jedoch in den Niederlanden.

Pathogene Y. enterocolitica-Stämme werden in Gewässern und Tieren, hier v.a. im Hausschwein, gefunden. Infektionen gehen von Lebensmitteln aus wie Milch und Milchprodukten sowie von Schweinefleisch [1], bevorzugt von den Innereien. Bei Infektionen konnte i.d.R. nachgewiesen werden, daß die Speisen nicht genügend durcherhitzt waren. Bei Ausbruchgeschehen ist mit einem großen Anteil asymptomatisch Infizierter zu rechnen [11]. Über nosokomiale Ausbrüche mit Übertragungen von Patient zu Patient ist berichtet worden (siehe [4]). Bei den Lebensmitteln ist von besonderer Bedeutung, daß die Kühllagerung ähnlich wie bei Listerien einen unzureichenden Schutz gegen das Keimwachstum bietet. Mit den meisten Infektionen ist in den Wintermonaten zu rechnen.

\subsection{Nachweismethoden und deren Aussagekraft}

Yersinien werden aus Stuhl, Darmbiopsie- bzw. Operationsmaterial, aus Blut und gelegentlich aus Material lokaler Herde angezüchtet. Zur Verwendung kommen meist primär selektive Nährböden, wie sie auch zum Nachweis von anderen Enterobacteriaceae eingesetzt werden. Spezifischere Nährböden sind der CIN- und der VYE-Nährboden, die eine sehr hohe Wiederfindungsrate erlauben und gleichzeitig den Kolonien ein sehr charakteristisches Aussehen verleihen. Mit ihrem primärem Einsatz kann i.d.R. nur gerechnet werden, wenn das Labor entsprechende Hinweise auf eine Yersinien-Infektion erhält. Seit lan- gem wird die Kälteanreicherung angewandt, die allerdings langwierig ist verglichen mit der Anzucht bei $37^{\circ} \mathrm{C}$ oder $25^{\circ} \mathrm{C}$. Bei $0-4^{\circ} \mathrm{C}$ haben Yersinien einen erheblichen Wachstumsvorteil gegenüber vielen anderen Begleitkeimen, die sich bei diesen Temperaturen z.T. überhaupt nicht vermehren. Neben Y.enterocolitica werden bei der Kälteanreicherung häufig auch generell als harmlos geltende Yersinien wie Y. fredericksenii, Y. intermedia, Y. kristeseii u.a. oder andere psychrophile Bakterien vermehrt.

Die Serotypisierung erfolgt durch Agglutination der Isolate mit spezifischen Antisera. Antiköpernachweis-Verfahren werden v.a. bei epidemiologischen Untersuchungen (Ausbruchanalysen) und bei Verdacht auf postinfektiöse Erkrankungen wie Arthritis, Myokarditis herangezogen. Ihr Einsatz im Spenderscreening ist abgesehen von damit verbundenen Kosten von zweifelhaftem Wert. Einmalige serologische Untersuchungen sind derzeit zum Nachweis frischer Infektionen ungeeignet. Darüber hinaus steht die sehr seltene Übertragung von yersinienhaltigen Konserven in deutlichem Kontrast zu der angegebenen Häufigkeit positiver serologischer Reaktionen. Heesemann und Karch [10] berichteten, daß bei Einsatz sog. universeller, d.h. serotypunabhängiger Yersinia-Antigene bei Blutspendern bis zu 40\% isoliert IgG-positiv und bis zu 9\% IgA-positiv gefunden wurden. Bei einem Spender können ansteigende Antikörpertiter in der Zeit nach der Spende als Zeichen einer frisch erworbenen Infektion gewertet werden und als Hinweis auf eine bakteriämische Phase zum Zeitpunkt der Spende gesehen werden. Derartige Einschätzungen sind jedoch mit großer Umsicht zu tätigen, da die Erfahrungen mit yersinienbedingten Transfusionszwischenfällen sehr gering sind.

\section{Blut und Plasmaspender}

\subsection{Prävalenz und Inzidenz bei Blutspenderkollektiven}

Eine Erhebung zur Häufigkeit von Spendewilligen, die Y. enterocolitica mit dem
Stuhl ausscheiden, ist nicht bekannt. Eine prospektive Studie zur Häufigkeit von Bakteriämien, ausgewiesen an bakteriologisch untersuchten, verfallenen Blutkonserven ist ebenfalls nicht bekannt. Dagegen berichten Heesemann und Karch [10] über die Häufigkeit von Spendern mit erhöhten Antikörpertitern gegenüber Y. enterocolitica. Wie bereits unter 1.4. ausgeführt, waren danach $40 \%$ isoliert IgG-positiv und bis $\mathrm{zu} 9 \%$ $\operatorname{IgA-positiv.~Eine~finnische~Studie~[12],~in~}$ der weniger spezifische Antigenpräparationen eingesetzt wurden, kam in der Gesamtbevölkerung auf eine Häufigkeit von bis zu $4 \%$ unter „unauffälligen“ Personen (vorheriger Ausschluß von reaktiven Arthritiden u.ä.).

\subsection{Spendertestung und Aussagekraft}

Eine routinemäßige Testung der Spender bzw. dessen Spende auf Bakterien im allgemeinen und $Y$. enterocolitica im speziellen wird nicht durchgeführt, da keine praktikable Methodik zur Verfügung steht. Beim Spender asymptomatisch oder symptomarm verlaufende Bakteriämien können somit unerkannt bleiben. Antikörpernachweise erscheinen ebenfalls nicht praktikabel. Der Nachweis einer frischen Infektion ist auf den Anstieg von spezifischem IgG angewiesen (s. 1.4), eine konsekutive, mindestens zweimalige serologische Untersuchung im Zusammenhang mit einer Blutspende wäre notwendig.

Genom-Nachweisverfahren (Gensonden, Nukleinsäure-Amplifikationsverfahren) sind derzeit nicht für einen derartigen Routineeinsatz geeignet. Von vereinzelt laufenden Untersuchungen unter Einsatz anderer Verfahren wird immer wieder berichtet.

\subsection{Spenderbefragung}

In einer Studie beim amerikanischen Roten Kreuz [13] wurde an ca. 11000 Spendewilligen überprüft, ob sie in den vergangenen vier Wochen Durchfall allein oder begleitet von ungewöhnlich starken Abdominalschmerzen und Fieber hatten. Letzteres bejahten $0,6 \%$ der Spender, Durchfall allein gaben $4 \%$ an. Daraus wurde geschlossen, daß eine Be- 
fragung ein relativ unspezifisches und auch ungenaues Mittel ist, um transfusionsassoziierte Übertragungen von Y. enterocolitica zu verhindern.

Von einem Blutspendedienst des Deutschen Roten Kreuzes wurde die Rückstellquote durch Durchfallanamnese an 335794 Spendewilligen ausgewertet. Spender, die im Fragebogen Durchfallerkrankungen in den letzten vier Wochen angaben, wurden nach vorgegebenen Regeln ausführlich befragt und anschließend eine ärztliche Entscheidung getroffen. Dadurch wurden 221 Spendewillige von der Blutspende zurückgestellt, was einem Anteil von o,066\% entspricht. Eine solche Rückstellquote wurde als unkritisch für die Blutversorgung eingestuft.

Aus Kanada wurde von einem Versuch berichtet, zum Zeitpunkt der Spende noch unerkannte Bakteriämien aufzudecken, indem man die Spender aufforderte, sich zu melden, wenn bei ihnen innerhalb der ersten Woche nach der Spende Durchfall oder eine Infektion aufträte. Etwa 0,03\% der Spender lösten eine Nachuntersuchung der Spende und ggf. Nachforschungen bei den Transfusionsempfängern aus. Weder waren Bakterien nachweisbar noch ließen sich Transfusionszwischenfälle eruieren [14]. Für Personen, die eine Eigenbluttransfusion vorbereiten, wird eine stringente Einhaltung der Kriterien, die für Fremdblutspenden gilt, empfohlen, um damit auch einer $Y$. enterocolitica-Übertragung vorzubeugen.

\subsection{Spenderinformation und -beratung}

Im Falle eines Transfusionszwischenfalles, der mit Y. enterocolitica in Zusammenhang gebracht wird, erfolgt gewöhnlich eine intensive Befragung des Spenders zur Aufklärung des epidemiologischen Zusammenhanges. Gegebenenfalls wird man ihn zu Nachuntersuchungen bitten, um in Stuhlproben nach dem Erreger zu suchen oder serologische oder andere Parameter für eine frisch erworbene Yersinien-Infektion $\mathrm{zu}$ überprüfen. Für den Spender selbst ist das Ereignis gewöhnlich ohne weitere Bedeutung, da bei ihm in den meisten
Fällen nur eine passagere Bakteriämie mit diesem Keim vorgelegen haben dürfte. Gelegentlich könnten sich aus dem Befund Hinweise auf bis dato unerklärte Gelenksbeschwerden ergeben.

\section{Empfänger}

\subsection{Prävalenz und Inzidenz von blutassoziierten Infektionen und Infektionskrankheiten bei Empfängerkollektiven}

In den USA sind den Gesundheitsbehörden von 1985 bis 1991 elf Fälle einer Yersiniensepsis im Zusammenhang mit der Transfusion von Erythrozytenkonzentraten berichtet worden, von 1991 bis 1996 zehn Fälle [8]. 50\% der durch Erythrozytenkonzentrate ausgelösten Sepsisfälle gingen in den USA auf Y. enterocolitica zurück [15]. Eine 1997 in den USA durchgeführte Risikobewertung kam zu dem Ergebnis, daß yersinienassoziierte Transfusionszwischenfälle sich mit einer Häufigkeit von etwa 1:500 000 übertragener Erythrozytenkonzentrate ereignen dürften. Eine ähnliche Einschätzung (1:1 Million) aufgrund britischer Zahlen trafen Mitchell und Barr [16]. Für Zwischenfälle mit tödlichem Ausgang findet sich in der Literatur die Angabe 1:9 Millionen [17], gestützt auf Zahlen, die der Food and Drug Administration, USA, gemeldet wurden. Es ist allerdings nicht bekannt, wieviele der tödlich verlaufenden Transfusionszwischenfälle der FDA tatsächlich bekannt werden. Möglicherweise würde eine systematischer durchgeführte bakterielle Untersuchung minder schwer verlaufender Transfusionszwischenfälle ebenfalls ein genaueres Abbild der Situation vermitteln. Die Übertragung von Yersinien durch gepoolte Thrombozytenkonzentrate ist bisher zweimal berichtet worden $[16,18]$. Bemerkenswert ist, daß in mehreren der berichteten Fälle die Transfusion von Thrombozytenkonzentraten unauffällig verlaufen war und Erythrozytenkonzentrate aus der gleichen Spende tödlich verlaufende Yersiniensepsen ausgelöst hatten [19-21].

Für Deutschland liegen keine zusammenfassenden Zahlen über einen ähnlichen Zeitraum vor. Kasuistische
Darstellungen von Zwischenfällen sind jedoch erschienen [22]; weiterhin liegt eine Meldung an das Paul-Ehrlich-Institut vor. Auch bei autologen Spenden sind Yersinia-Sepsis-Fälle wiederholt berichtet worden [23, 24].

Auffallenderweise wurden bei den Transfusionszwischenfällen mit Y. enterocolitica weltweit nur Stämme identifiziert, die keine Siderophorenbildung aufweisen, nämlich die Serovare O:3, O:9, O:5,27 usw. (siehe dazu die Aufstellungen bei $[4,7,21])$. Der in den USA relativ weit verbreitete und allgemein als virulenter angesehene siderophorenbildende Serovar O:8 wurde auch dort bisher nicht bei Transfusionszwischenfällen beobachtet (siehe hierzu auch 1.1.).

Die bisherigen Kenntnisse sprechen dafür, daß durch Y. enterocolitica bedingte Transfusionszwischenfälle vor 1985 deutlich seltener gewesen sein müssen als in den Jahren danach. Auf der Suche nach relevanten Veränderungen, die Mitte der achtziger Jahre eingetreten waren und möglicherweise die erhöhte Zahl an Zwischenfällen erklären könnten, konzentrierten sich Gibb et al. [25] auf die Einführung der additiven Lösung zur Konservierung der Erythrozyten. In den USA erfolgte dies beispielsweise zwischen 1983 und 1985. Die additive Lösung ersetzt das zuvor verwendete Plasma bis zu 90\%. Gibb et al. zeigten, daß bereits in Plasmakonzentrationen unter $25 \%$, anders als in Vollplasma, komplementempfindliche Y. enterocolitica nicht mehr abgetötet werden. Es wäre zu überprüfen, ob im gleichen Zeitraum auch das Intervall zwischen der Spende und der Komponententrennung verkürzt worden ist. Damit wäre die vor dem Plasmaersatz verbleibende Zeit einer wirksamen Komplementeinwirkung vermindert.

Als dritte zu diskutierende Möglichkeit bringen Gibb et al. noch eine angestiegene Frequenz an Y. enterocolitica-Infektionen in der Gesamtbevölkerung während der achtziger Jahre ins Spiel. Dafür gibt es weltweit keine Hinweise. Interessanterweise wurden aus Belgien, wo sich die Isolate an Y. enterocolitica in der Gesamtbevölkerung von Mitte der achtziger Jahre bis 1996 um das Sechsfache verminderten [1], keine 
auffallenden Veränderungen bei Transfusionszwischenfällen berichtet. In Neuseeland hingegen, wo zwischen 1991 und 1996 acht Y. enterocolitica-assoziierte Transfusionszwischenfälle (davon fünf mit tödlichem Ausgang) beobachtet worden waren, welches einer Häufigkeit von 1:65 000 entspricht, sind gesamtepidemiologische Überlegungen angestellt worden [21].

\subsection{Abwehrlage (Resistenz, vorhandene Immunität, Immunreaktivität, Alter, exogene Faktoren)}

Die Transfusion einer mit Bakterien hochkontaminierten Blutkonserve ist immer lebensbedrohlich. Die Keime können eine Dichte von $10^{9} / \mathrm{ml}$ erreichen. Noch gravierender wiegt der Gehalt an Endotoxin, das von den Keimen bei der Vermehrung freigesetzt wird. In transfundierten Erythrozytenkonzentraten wurden Konzentrationen von 600 $\mathrm{ng} / \mathrm{ml}$ wiederholt gemessen. Endotoxin in der Menge von $\geq \mathrm{ng} / \mathrm{kg}$ Körpergewicht gilt bereits als ausreichend für die Auslösung einer fieberhaften Reaktion (siehe [26]). Wenige Milliliter einer Konserve können daher schon ausreichen, den Patienten in Lebensgefahr zu bringen. Dies entspricht auch den in Kasuistiken wiedergegebenem klinischen $\mathrm{Ab}$ lauf.

Weder die spezifische Abwehr, die sich infolge einer vorausgegangenen Infektion mit $Y$. enterocolitica ausgebildet haben mag, noch die unspezifische $\mathrm{Ab}$ wehr bieten einen Schutz gegen die Überschwemmung der Blutbahn mit Bakterien und Endotoxin. Man muß bei Exposition davon ausgehen, daß gegebenenfalls vorhandene spezifische Antikörper binnen kurzem aufgebraucht wären. Die Tage beanspruchende Boosterreaktion ist gegenüber dem akuten Ereignis ohne Belang. Bei geringeren Konzentrationen an Bakterien und Endotoxin mag die allgemeine Konstitution, einschließlich des Alters, bei der Ausprägung der Schocksymptome von Bedeutung sein. Untersuchungen dazu liegen nicht vor.

\subsection{Schwere und Verlauf der Erkrankung}

Bei den zehn in den USA für den Zeitraum 1991-1996 belegten Y. enterocolitica-Zwischenfällen starben fünf Patienten innerhalb von sechs Tagen nach der Transfusion. Die Transfusion einer Yersinien-belasteten Konserve löst die für einen Endotoxinschock typischen Symptome wie Kreislaufzusammenbruch, Fieber, Atemnot (ARDS) und disseminierte intravaskuläre Gerinnung (DIC) aus. Unter minder belasteten Konserven kann sich das klinische Bild zunächst nur auf Hypotonie und erhöhte Temperaturen beschränken. Ein tödlicher Ausgang, der trotz intensivmedizinischer Betreuung eintritt, beruht meist auf Multiorganversagen.

Wenn eine belastete Konserve unter Narkose transfundiert wird, können die ersten Zeichen der Endotoxinwirkung, ansteigende Temperatur und Blutdruckabfall, kaschiert sein oder nur sehr diskret auftreten.

\subsection{Therapie- und Prophylaxemöglichkeiten}

Antibiotika helfen nicht gegen die akuten Auswirkungen der Yersiniensepsis. Die Patienten bedürfen des sofortigen intensivmedizinischen Einsatzes zur Bekämpfung des Endotoxinschocks. Jedoch muß durch eine zu diesem Zeitpunkt breit angelegte Antibiose - der Erreger ist gewöhnlich nicht bekannt - die Vermehrung der infundierten Erreger verhindert werden. Inwieweit hierbei zu berücksichtigen ist, daß aus den dann zerfallenden Erregern weiteres Endotoxin freigesetzt wird, ist in der Literatur noch nicht eingehend diskutiert worden (Vergl. [27]). Wie grundsätzlich bei jedem Transfusionszwischenfall sollte noch vor Beginn der Antibiotikaapplikation eine Blutkultur angelegt werden, um den noch unbekannten Erreger identifizieren zu können. Das läßt sich nicht immer verwirklichen, da der Patient bereits aus anderen Indikationen mit Antibiotika behandelt sein kann. Auch dann jedoch sollte die Anlage einer Blutkultur nicht unterbleiben, der Umstand der Antibiotikagabe unter Angabe des Präparates aber bei der
Weitergabe des Untersuchungsmaterials vermerkt werden. Die Konservenbeutel bzw. seine Ausgänge sind aseptisch zu verschließen und umgehend der ausgebenden Transfusionszentrale zuzuleiten, da dort oder von dort aus die notwendigen Maßnahmen, einschließlich der mikrobiologischen Untersuchungen, veranlaßt werden müssen. Es ist sinnvoll, über die übliche mikrobiologische Diagnostik hinaus eine Keimzahlbestimmung sowie einen Pyrogennachweis vorzunehmen.

Unmittelbare prophylaktische Maßnahmen, die Auswirkungen einer möglicherweise kontaminierten Konserve abwehren könnten, sind nicht bekannt. Eine antibiotische Prophylaxe allein unter dieser Maßgabe ist nicht angezeigt, da sie, wie oben ausgeführt, gegen den Endotoxinschock unwirksam ist. In nicht wenigen Fällen dürften Transfusionen allerdings unter antibiotischer Behandlung erfolgen, die vom Grundleiden oder perioperativ bestimmt ist. Nach einer überstandenen Sepsis können sich metastatische Herde (s. 1.2) entwickeln. Dieser Gefahr muß ggf. durch eine angepaßte länger dauernde antibiotische Behandlung begegnet werden.

\section{5 Übertragbarkeit}

Nach einer überstandenen Yersiniensepsis dürfte von dem Patienten nach dem gegenwärtigen Kenntnisstand keine Gefahr für seine nächste Umgebung ausgehen. Es ist nicht bekannt, inwieweit mit andauernder Ausscheidung im Gefolge $\mathrm{zu}$ rechnen ist, und ob eine solche eine gesundheitliche Gefahr für ihn oder seine Umgebung bedeuten könnte. Zu den Sequelae einer Infektion mit Y. enterocolitica wie z.B. Arthritis siehe unter 1.2.

\subsection{Häufigkeit und Menge der Applikation von Blutprodukten}

Eine Belastung mit Y. enterocolitica ist nur bei Erythrozytenkonzentraten und in weit geringerem Umfang bei Thrombozytenkonzentraten zu erwarten. Dabei macht es keinen Unterschied, ob es sich um Konserven aus Fremd- oder Eigenblutspenden handelt. Das Risiko, mit der Erythrozytenkonzentrate belastet sein dürften, kann unter Vorbehalt bei 
1:500 000 angegeben werden (s. dazu die weiteren Ausführungen in 3.1). Bei gefrorenem Frischplasma oder Plasmaderivaten ist nicht mit einer massenweisen Übertragung von $Y$. enterocolitica $\mathrm{zu}$ rechnen.

\section{Blutprodukte}

\subsection{Belastung des Ausgangsmaterials und Testmethoden}

In der Regel ist von sehr geringen Keimkonzentrationen kurz nach der Spende auszugehen, auch wenn schließlich $>10^{9}$ Keime pro $\mathrm{ml}$ bei Transfusionszwischenfällen gefunden werden. Nach Modellversuchen mit inokulierten Erythrozytenkonzentraten reichen ein bis zehn Keime pro ml für den Start einer derartigen Vermehrung aus. Tatsächlich in einer Spende aufgetretene initiale Konzentrationen sind bisher jedoch noch nicht bestimmt worden. Für die Annahme einer sehr niedrigen initialen Keimkonzentration spricht auch die Beobachtung, daß in unmittelbar nach der Spende abgetrennten Schlauchabschnitten yersinienbelasteter Konserven $\left(10^{7}-10^{9}\right.$ $\mathrm{CFU} / \mathrm{ml}$ ), die zu schwersten Zwischenfällen führten, keine Keime nachweisbar waren [28].

Praktikable Verfahren zur routinemäßigen Überprüfung jeder Spende, wie sie bei viralen Markern üblich sind, stehen nicht zur Verfügung. Die Sterilitätstestung in den Blutspendediensten arbeitet mit Stichproben und dient allein dem Erfordernis, zur Qualität der Konservenzubereitung und ggf. der der Spender Aussagen zu treffen. Sie eignet sich nicht dazu, belastete Konserven aussondern zu wollen. Die initial als sehr niedrig vermutete Konzentration der Keime dürfte häufig unter der Nachweisgrenze einer Sterilitätstestung bzw. einer NAT liegen. Demzufolge könnte ein Test erst nach Vermehrung der Keime (z.B. vor der Ausgabe des Präparates) erfolgreich eingesetzt werden. Ein solches Vorgehen würde jedoch die Ausgabe der benötigten Blutkonserve gegenwärtig in nicht hinnehmbarer Weise verzögern, da der Zeitbedarf für die kulturellen Techniken bzw. eine NAT zu hoch ist. Hinzu kommt, daß für solche Tests der Beutel geöffnet werden müßte, was mit Kontaminationsrisiken verbunden und unbedingt $\mathrm{zu}$ vermeiden ist. Schlauchabschnitte, die ein Volumen von 300 bis $500 \mu \mathrm{l}$ aufweisen, eignen sich, wie oben ausgeführt, dazu nicht. Unabhängig von der klinischen Erfahrung ist bei den vermuteten niedrigen Konzentrationen von ein bis zehn Keimen pro $\mathrm{ml}$ allein schon aus statistischen Gründen mit erregerfreien Segmenten zu rechnen.

Für weniger aufwendige Verfahren anstelle der Erfassung einer Keimvermehrung wie z.B. die Verwendung von PCR auf Yersinia enterocolitica liegen Ergebnisse vor [29]; sie sind jedoch für den routinemäßigen Einsatz nicht geeignet. Andere direkte Bestimmungsverfahren wie Gramfärbung, Akridinorangefärbung, universelle Gensonden auf bakterielle rRNA werden als zu insensitiv bzw. wenig praktikabel eingeschätzt [21]. Indirekt ansetzende Verfahren (Surrogatteste) wie die Erfassung von $\mathrm{pH}$-Wert-Veränderungen oder der verminderte Glukosegehalt der Konserve sind verschiedentlich vorgeschlagen worden. Sie wären unter vergleichsweise geringem zeitlichem und technischen Aufwand einsetzbar. Bei artifiziell inokulierten Thrombozytenkonzentraten zeigten Urin-dipsticks erniedrigte $\mathrm{pH}$ - und Glukosewerte an [30]. Unter den überprüften Enterobacteriaceae waren Klebsiella pneumoniae und Serratia marcescens, jedoch nicht Yersinia enterocolitica. Das Verfahren ist gegenwärtig bei klinisch eingesetzten Thrombozytenkonzentraten in der Erprobung. Über einen Versuch, das Verfahren auf Erythrozytenkonzentrate auszudehnen, wurde bisher nicht berichtet. Es wäre unmittelbar vor der vorgesehenen Transfusion sinnvollerweise einzusetzen. Die Öffnung des Beutels wäre dann sowieso notwendig, und es käme dabei die für die Transfusion maßgebende Keimkonzentration zum Tragen.

Aus der Literatur ist bekannt, daß selbst hochgradig kontaminierte Konserven äußerlich unauffällig waren. Lediglich in einem (tödlich verlaufenen) Fall wird von einem hämolytisch veränderten Plasma des Konservenrestes be- richtet [28]. Daher kann man sich nicht auf die immer wieder berichteten Zeichen einer mikrobiologischen Besiedlung wie dunkle Verfärbung, Klumpenbildung, Hämolyse oder Verfärbung des (zellfreien) Überstandes stützen, um die Transfusion einer mit Y. enterocolitica kontaminierten Konserve vermeiden. Das Gebot, die Konserven vor der Transfusion auf die genannten Zeichen hin sorgfältig zu inspizieren, bleibt davon jedoch unberührt.

\subsection{Möglichkeit der Abtrennung und Inaktivierung von Infektionserregern}

Als umfassende Maßnahme gegen ein bakterielles Wachstum in den Blutbeuteln ist vorgeschlagen worden, die Konserven mit Antibiotika zu versetzen [31]. Die Verteuerung der Konserven und die zu befürchtenden Nebenwirkungen wie Allergisierung bzw. das Vorliegen einer Allergie bei den Empfängern und eine Förderung der Resistenzentwicklung hielten von einer solchen Maßnahme ab.

Wie experimentell an den sehr selten mit Y. enterocolitica kontaminierten Thrombozytenkonzentraten gezeigt wurde, ist eine Abtötung dieser (und anderer) Erreger in Blutkomponenten grundsätzlich möglich. Durch Behandlung mit einem Psoralen (S-59) und anschließender UV-Bestrahlung (UVA, 320-400 nm) konnte Y. enterocolitica um den Faktor $10^{6}$ reduziert werden [32]. Für die typischerweise betroffenen Erythrozytenkonzentrate liegen keine derartigen Experimente vor. Ohnehin müßte zunächst geklärt werden, ob eine solche Behandlung die Erythrozyten schädigt und toxikologisch unbedenklich ist. Weiterhin ist die Lichtdurchlässigkeit dieser Blutkomponenten in der gegenwärtig benutzten Beutelabfüllung unzureichend.

In vielfältigen experimentellen Studien wurde überprüft, ob Y. enterocolitica mittels Leukozytenfiltration zu entfernen sei. Generell ist (bei Unterschieden zwischen den Serotypen) eine wesentliche Reduktion bis hin zur Eliminierung der Bakterien beobachtet worden. Die überwiegende Mehrzahl dieser Untersuchungen leidet jedoch unter methodischen Mängeln (z.B. fehlender 
Nachweis von Antikörpern gegen Y. enterocolitica, Nichtberücksichtigung eventuellen Verlustes von Virulenzplasmiden während der Anzucht im Labor, Vernachlässigung der Unterschiede zwischen den verschiedenen Serotypen), so daß ihre Interpretation nur unter Vorbehalt möglich ist. Bei zusammenfassender Wertung aller experimenteller Daten kann jedoch festgestellt werden, daß es zu einer zeitabhängigen Verminderung der Zahl vermehrungsfähiger Y. enterocolitica durch die Aufbewahrung der (Vollblut-)Spende bei Raumtemperatur kommt (siehe hierzu auch 1.1. Erregereigenschaften). Diese Bewertung dieser Ergebnisse ging mit der Vorstellung einher, daß die Keime als fakultativ-intrazelluläre Bakterien vorwiegend in Phagozyten (Monozyten, Granulozyten) überleben oder mit ihnen auch nur assoziiert sind, so daß Leukozytendepletion zur Reduktion der Bakterien führt. Die Lagerung selbst läßt die bakteriziden Elemente des Plasmas zum Zuge kommen (s. unten und auch die ausführliche Darstellung bei [33]).

Festlegungen zur optimalen Lagerungszeit des Vollblutes vor der Leukozytenfiltration sind in der Literatur nicht zu finden. Buchholz et al. [9] hatten in ihren experimentellen Untersuchungen mit Y. enterocolitica O:3 durchweg mit einer Verweilzeit von sieben Stunden Raumtemperatur gearbeitet. Sie hatten damit unter Ausnahme der höchsten Erregerbelastung (132 CFU/ml) komplette Sterilität der Proben nach Leukozytenfiltration demonstrieren können.

Die Bedeutung des Komplementsystems für die Abtötung von Y. enterocolitica in der Vollblutspende bzw. in den Erythrozytenkonzentraten wurde von Gibb et al. [25] untersucht. Sie wiesen mit präzis definierten Stämmen nach, $\mathrm{daß}$ ab einer Plasmakonzentration unter $26 \%$ komplementempfindliche Y. enterocolitica nicht mehr zuverlässig vom Komplement abgetötet werden können. Aus ihren Ergebnissen folgerten sie, daß die durch die Komponententrennung bewirkte Plasmaverdünnung frühestens sechs Stunden nach Lagerung bei Raumtemperatur erfolgen dürfe, um alle extrazellulären Keime zu eliminieren. Aus den experimentellen Vorgaben von
Buchholz et al. [9] und Gibb et al. [25] kann die Empfehlung abgeleitet werden, Spenden unfraktioniert für mindestens sechs Stunden bei Raumtemperatur zu lagern, bevor man sie in die Komponenten auftrennt.

\subsection{Praktikabilität und Validierbarkeit der Verfahren zur Elimination/ Inaktivierung von Infektionserregern}

Während Yersinia enterocolitica die Sicherheit von Blutkomponenten erheblich beeinträchtigen kann, spielen sie bei Plasmaderivaten keine Rolle. Typischerweise sind Erythrozytenkonzentrate betroffen, in Ausnahmen Thrombozytenkonzentrate; über Zwischenfälle mit gefrorenem Frischplasma (GFP) liegen keine Berichte vor. Elimination bzw. Inaktivierung von Bakterien bzw. der Yersinien in zellulären Blutzubereitungen sind noch keine praktizierten Verfahren. Möglicherweise ließen sich mit der Leukozytenfiltration, die u.a. zur Elimination intrazellulärer Viren sowie des Creutzfeldt-Jakob-Agens propagiert wird, auch ggf. vorhandene Yersinien entfernen. Offen bleibt, ob die optimal erscheinenden Bedingungen zur Abtrennung einiger Bakterienspezies und insbesondere der Yersinien mit anderen Anforderungen an die Aufbereitung der Vollblutspende vereinbar sind. Ob eine Leukozytendepletion tatsächlich eine reale Verbesserung darstellt, könnte erst nach einer langen Beobachtungszeit festgestellt werden. Die bisher beobachteten geringen Fallzahlen erlauben keine kurzfristige Bewertung.

\section{Bewertung}

Durch Y. enterocolitica bedingte Transfusionsreaktionen sind seltene Ereignisse. Die Gesamtzahl der durch Y. enterocolitica ausgelösten Transfusionsreaktionen wird mit 1:500 ooo angegeben. Für tödlich verlaufende Zwischenfälle werden in der Literatur Häufigkeiten zwischen 1:1 Mio bis 1:10 Mio berichtet. Beim Umgang mit diesen Zahlen ist jedoch zu beachten, daß bislang die systematische Erfassung bakterieller Transfusionszwischenfälle vernachlässigt wurde. Aktuelle Studien, z.B. die derzeit in den USA laufende Bacony Studie der CDC oder die Hämovigilanzerhebungen der Agence Française du Sangue, sollen Daten hierzu erbringen. Von entscheidender Bedeutung ist es, bei Transfusionsreaktionen die Möglichkeit einer Y. enterocolitica-Infektion in Betracht $\mathrm{zu}$ ziehen, gegebenenfalls diagnostische Schritte einzuleiten und eine Meldung zu veranlassen.

Die Abwägung von denkbaren prophylaktischen Maßnahmen, die seit Ende der achtziger Jahre diskutiert werden, ist schwierig, da die Inzidenzen niedrig und die Basiszahlen zur Ausgangslage dürftig sind. Solche Voraussetzungen erschweren eine sachgerechte Kosten-Nutzen-Analyse, speziell für eine allein auf die Yersinien ausgerichtete Maßnahme.

Verschiedentlich wird vorgeschlagen, Spender auszuschließen, die in den vorausgegangenen vier Wochen fieberhafte Erkrankungen und/oder Durchfallerkrankungen hatten. Vorliegende Daten aus Deutschland sprechen dafür, daß die Rückstellrate von Spendern nicht wesentlich ansteigen würde. Aufgrund der spärlichen Datenlage ist zwar nicht zu belegen, daß eine solche Befragung zur Verminderung der Transfusionsreaktionen durch $Y$. enterocolitica führt. Es ist jedoch eine Reduktion von Kontaminationen durch Y. enterocolitica und andere bakterielle Erreger zu erwarten. Es wird daher empfohlen, eine Frage nach fieberhaften Erkrankungen und/oder Durchfallerkrankungen in die Spenderauswahlkriterien aufzunehmen.

Viele Gesichtspunkte sprechen für die Leukozytenfiltration, wenn sie nach ausreichender Vorinkubation der unfraktionierten Spende bei Raumtemperatur erfolgt. Inzwischen ist die Leukozytenfiltration in einigen Ländern Europas aus anderen Gründen eingeführt; in anderen Ländern, wie z.B. Deutschland, wird ihre Einführung in Betracht gezogen. Man sollte diese Entwicklung nutzen und versuchen, die Bedingungen, unter denen dieses Verfahren gehandhabt wird, so zu gestalten, daß dabei auch Yersinien entfernt werden können (d.h. eine Lagerung des Vollbluts für mindestens sechs Stunden bei Raumtemperatur vor Leukofiltration). 
Dieses Papier wurde fertiggestellt am 23.2.1998 und vom Arbeitskreis Blut am 3.5.1999 verabschiedet. Es wurde erarbeitet von den Mitgliedern der Untergruppe „Bewertung Blut-assoziierter Krankheitserreger" des Arbeitskreises Blut: Prof. Dr. Reinhard Burger, Prof. Dr. Wolfram Gerlich, Prof. Dr. Lutz Gürtler, Dr. Margarethe Heiden, Prof. Dr. Bernd Jansen, Prof. Dr. Volker Kretschmer, Dr. Hans Lefèvre, PD Dr. Johannes Löwer, Dr. Thomas Montag-Lessing, PD Dr. Rainer Neumann, Prof. Dr. Georg Pauli, Prof. Dr. Rainer Seitz, Dipl. Med. Uwe Schlenkrich, Dr. Edgar Werner, Dr. Hannelore Willkommen.

\section{Literatur}

1. Verhaegen J, Charlier J, Lemmens P, et al. (1998) Surveillance of human Yersinia enterocolitica infections in Belgium: 1967-1996. Clin Infect Dis 27:59-64

2. Heesemann J (1994) Die Gattung Yersinia, Yersiniosen. In: Brandis H, Eggers HJ, Köhler W, Pulverer G (Hrsg) Lehrbuch der Medizinischen Mikrobiologie (7.Aufl.). Gustav Fischer, Stuttgart

3. Bradley RM, Gander RM, Patel SK, Kaplan HS (1997) Inhibitory effect of $0^{\circ} \mathrm{C}$ storage on the proliferation of Yersinia enterocolitica in donated blood. Transf 37:691-695

4. Bottone EJ (1997) Yersinia enterocolitica: The charisma continues. Clin Microbiol Rev 10:257-276

5. Jurado RL (1997) Iron, infections, and anemia of inflammation. Clin Infect Dis 25 : 888-895

6. Hoogkamp-Korstanje JAA, de Koning J, Heesemann J (1998) Persistence of Yersinia enterocolitica in man. Infection 16:81-85

7. Högman CF, Engstrand L (1996) Factors affecting growth of Yersinia enterocolitica in cellular blood products. Transf Med Reviews 10:259-275
8. US Dept of Health and Human Services, Centers for Disease Control and Prevention (1997) Red blood cell transfusions contaminated with Yersinia enterocolitica United States, 1991-1996, and initiation of a national study to detect bacteriaassociated transfusion reactions. Morbidity and Mortality Weekly Report 46:553-555

9. Buchholz DH, AuBuchon JP, Snyder EL, Kandler R, Edberg S, Piscitelli V, Pickard C, Napychank P (1992) Removal of Yersinia enterocolitica from AS-1 red cells. Transf 32:667-672

10. Heesemann J, Karch H (1995) Diagnostik von Yersiniosen und Infektionen mit enterohämorrhagischen Escherichia coli (EHEC). Internist 36: 102-105

11. Van Ossel C, Wauters G (1990) Asymptomatic Yersinia enterocolitica infections during an outbreak in a day-nursery. Eur $\mathrm{J} \mathrm{Clin}$ Microbiol Infec Dis 9: 148-149

12. Granfors K, Isomäki H.von Essen R, Maatela J, Kalliomäki JL, Toivanen A (1983) Yersinia antibodies in inflammatory joint disease. Clin Exp Rheumatol 1:424-429

13. Grossman BJ, Kollins P, Lau PM, Perreten JL, Bowman RJ, Malcolm S, Palko WM (1991) Screening blood donors for gastrointestinal illness: a strategy to eliminate carriers of Yersinia enterocolitica. Transf 31: 500-501

14. Goldman M, Long A, Roy G, et al. (1995) Incidence of positive bacterial cultures after donor call-back (abstract). Transf 35 [Suppl]:34

15. Klein HG, Dodd RY, Ness PM, Fratantoni JA, Nemo GJ (1997) Current status of microbial contamination of blood components: summary of a conference. Transf 37:95-101

16. Mitchell R, Barr A (1992) Transfusing Yersinia enterocolitica. Brit Med J 305: 1095-1096

17. Prentice $M(1992)$ Transfusing Yersinia enterocolitica. Brit Med J 305:664-665

18. Kuehnert MJ, Jarvis WR, Schaffer DA, Chaffin DJ (1997) Platelet transfusion reaction due to Yersinia enterocolitica. JAMA 278:550

19. Stubbs JR, Ramakrishna LR, Elg SA, et al. (1991) Fatal Yersinia enterocolitica (Serotype $0: 5,27)$ sepsis after blood transfusion. Vox Sang 61:18-23

20. McDonald CP, Barbara JAJ, Hewitt PE, et al. (1996) Yersinia enterocolitica transmission from a red cell unit 34 days old. Transf Med 6:61-63

21. Theakston EP,Morris AJ, Streat SJ, Baker BW, Woodfield DG (1997) Transfusion transmitted Yersinia enterocolitica infection in New Zealand. Aust NZ J Med 27:62-67
22. Schwalbe B, Kneitinger E, Marre R, Kubanek B, Gottfried HW, Georgieff M (1996) Letale Yersiniensepsis nach intraoperativer Transfusion. Anasthesiol Intensivmed Notfallmed Schmerzther 31:658-660

23. Haditsch $M$, Binder L, Gabriel C, Müller-Uri P, Watschinger R, Mittermayer H (1994) Yersinia enterocolitica septicemia in autologous blood transfusion. Transf 34:907-909

24. Richards C, Kolins J, Trindade CD (1992) Autologous transfusion-transmitted Yersinia enterocolitica. JAMA 268:1541-1542

25. Gibb AP, Poling N, Murphy WG (1996) Failure to kill Yersinia enterocolitica by plasma diluted to the concentration found in red cell units. J Clin Pathol 49:434-436

26. Arduino MJ, Bland LA, Tipple MA, et al (1989) Growth and endotoxin production of Yersinia enterocolitica and Enterobacter agglomerans in packed erythrocytes. J Clin Microbiol 27:1483-1485

27. Holzheimer RG (1998) The significance of endotoxin release in experimental and clinical sepsis in surgical patients - evidence for antibiotic-induced endotoxin release? Infection (Germany) 26:77-84

28. Tipple MA, Bland LA, Murphy JJ, Arduino MJ, Panlilio AL, Farmer III JJ, Tourault MA, et al. (1990) Sepsis associated with transfusion of red cells contaminated with Yersinia enterocolitica. Transf 30:207-213

29. Feng P, Keasler SP, Hill WE (1992) Direct identification of Yersinia enterocolitica in blood by polymerase chain reaction amplification. Transf 32:850-854

30. Burstain JM, Brecher ME, Workman K, Foster M, Faber GH, Mair D (1997) Rapid identification of bacterially contaminated platelets using reagent strips: glucose and $\mathrm{pH}$ analysis as markers of bacterial metabolism. Transf 37:255-258

31. Wagner SJ, Friedman LI, Dodd RY (1994) Transfusion-associated bacterial sepsis. Clin Microbiol Rev 7:290-302

32. Lin L, Cook DN, Wiesehahn GP, Alfonso R, Behrman B, et al. (1997) Photochemical inactivation of viruses and bacteria in platelet concentrates by use of a novel psoralen and long-wavelength ultraviolet light. Transf 37: 423-435

33. Dzik W (1995) Use of leukodepletion filters for the removal of bacteria. Immunol Invest 24: $95-115$ 